Instituto Internacional de Investigación y Desarrollo Tecnológico Educativo INDTEC, C.A.

DOI: https://doi.org/10.29394/Scientific.issn.2542-2987.2018.3.7.2.37-56

OAI-PMH: http://www.indteca.com/ojs/index.php/Revista Scientific/oai

\title{
Estrategias Constructivistas Aplicadas por el Docente para el Aprendizaje de la Física en el Nivel Superior
}

\author{
Autor: Avilner Rafael Páez Pereira \\ Universidad Nacional Experimental "Rafael María Baralt", UNERMB \\ profavilnerp@gmail.com \\ Zulia, Venezuela
}

\section{Resumen}

El objetivo de este estudio consistió en determinar las estrategias constructivistas aplicadas por el docente para el aprendizaje de la física en la carrera Educación Matemática y Física en la Universidad Nacional Experimental "Rafael María Baralt". La investigación fue de tipo descriptiva, con diseño de campo, documental, prospectiva y transversal. Se utilizaron como instrumentos un cuestionario de respuestas policotómicas de 34 ítems dirigido a los docentes, así como una prueba objetiva de preguntas cerradas de selección simple con 21 ítems, destinada a los estudiantes. Los resultados obtenidos evidencian que los profesores, no aplican en su mayoría, las estrategias constructivistas más adecuadas para lograr en sus estudiantes aprendizajes significativos, puesto que en un $60 \%$ los educandos no han alcanzado una formación idónea sobre la disciplina, además que los facilitadores frecuentemente no consideran, estrategias para el procesamiento de la información, el contexto donde se van a desarrollar las mismas y los estilos de construcción del conocimiento del aprendiz.

Palabras clave: estrategias educativas; aprendizaje; física. 


\title{
Constructive Strategies Applied for the Teacher to the Learning of the Physic in the Superior Level
}

\begin{abstract}
The objective of this study consisted in to determinate the constructive strategies applied for the teacher to the learning of the physic in the course Education mathematic and physic in the Experimental National University "Rafael María Baralt". The investigation was descriptive with design of camp, documental, prospective and transversal. It was used as instruments a questionnaire of several answers of 34 items for the teachers as soon as an objective test with closed questions of simple selection with 21 items for the students. The resulting gotten show that the teachers don't apply, in the main, the constructive strategies most adapted to obtain in their students significant learning, since in a $60 \%$ the pupils haven't reached an ideal formation in the discipline, also the facilitators frequently don't consider strategies to the information processing, the context where it goes to development this and the stiles of construction of the knowledge of the learner.
\end{abstract}

Keywords: educational strategies; learning; physics. 


\section{Introducción}

Las nuevas tendencias para la enseñanza de la Física en el país demandan de la aplicación de estrategias innovadoras enfocadas en corrientes como el constructivismo. Esta corriente ofrece alternativas al método tradicional de enseñanza, buscando la interactividad con el proceso que se quiere analizar y tomando en cuenta otros factores relacionados con el ambiente social y la forma en cómo se aprende, siendo en el aprendizaje de la física, relevante la formación que requiere el docente que imparte la materia.

En conformidad con lo anterior, el presente trabajo de investigación, tiene como objetivo general determinar las estrategias constructivistas aplicadas por el docente para el aprendizaje de la física en la carrera Educación Matemática y Física, en la Universidad Nacional Experimental "Rafael María Baralt", puntualizando en las formas de enseñanza de la asignatura en el nivel de licenciatura, y por consiguiente describiendo los aspectos a tener en cuenta para lograr un mejor rendimiento en ésta, así como los métodos formativos y los estilos de aprendizaje existentes, para posteriormente plantear diversas alternativas para la solución de posibles problemáticas.

Por otra parte, es importante destacar que el proceso de enseñanza aprendizaje de ciencias como la física siempre es un tema de preocupación y gran significación en cualquiera de los niveles educativos, desde los básicos hasta los cursos más avanzados donde ésta se imparte, ya sea en su esencia pura o como complemento de otras carreras. Esto se debe a que esta ciencia además de desarrollar principios conceptuales mediante el proceso de experimentación, también toma como base muchos de los conceptos, signos y representaciones propios de las matemáticas, los cuales tienen que resultar más afines al educando para emplearlos o transferirlos a las situaciones estudiadas en esta asignatura, así como nociones generales de la ciencia y hasta del lenguaje común, a los que en la mayoría de los casos debe atribuirle 
diferente significado al conocido hasta ese momento.

En ese sentido, considerando los planteamientos de Llamas (2008a: 14), para el aprendizaje de la física se requiere de un proceder didáctico el cual no puede ser el clásico memorístico y entre las exigencias para el estudio de la asignatura debe dársele gran importancia a los pasos que han de seguirse para la formación y desarrollo del pensamiento teórico, sobre cuya base se construyen los conceptos científicos, obligándose al docente a prepararse en función de satisfacer las necesidades de conocimientos solicitados por sus estudiantes y aplicando las diversas técnicas y estrategias de enseñanza acordes con el contexto social predominante.

\section{EI Problema}

La enseñanza de las ciencias y la profesionalización de los docentes en estas áreas, en todos los niveles del sistema educativo, del universitario al magisterial, según Soussan (2009: 5), es un tema de gran significación a nivel mundial, generando preocupación aun en los países de mayor desarrollo. Esto, debido a que ciencias como las matemáticas o la física presentan ciertos grados de abstracticidad o experimentación los cuales dificultan su aprendizaje, siendo su dominio esencial en muchos de los campos de estudios superiores.

En este orden de ideas, Braga, Gallardo, Calderón, Morales, y Kling (2011a: 5), manifiestan que cuando se estudia física, el educando debe estar en la capacidad de aplicar representaciones de su realidad entre las cuales nombran el uso necesario de símbolos, modelos, diagramas, afirmaciones verbales, así como las fórmulas y los gráficos. Sin embargo, de acuerdo con los autores, más del $40 \%$ de los participantes que ingresan a la Universidad tienen problemas al momento de establecer conexión entre la realidad y sus diversas formas de representación, sin dejar de mencionar que existe en más del $70 \%$ de estos, una predisposición a la constante memorización de ciertos 
fenómenos o sucesos, sin dedicar un espacio a la comprensión de los mismos, persistiendo esta situación durante la mayor parte del proceso de formativo.

De esta forma, el mencionado autor plantea, en los cursos de Física General de nivel universitario, la suposición es que los educandos han desarrollado una gama de conocimientos previos partiendo de las situaciones cotidianas, no obstante, la realidad parece ser otra puesto que la mayoría de los ingresados en la carrera denotan un bajo nivel de conexión entre teoría y práctica, lo cual se refleja en la incapacidad para abstraer fenómenos reales, y en consecuencia se les dificulta el trabajo al momento de manipular conceptos abstractos, los cuales resultan ser necesarios para el desarrollo adecuado del proceso de enseñanza aprendizaje en un área de ciencia con ciertos niveles de complejidad como lo es la física.

Entonces, quizás los estudiantes en el nivel superior presentan algunas deficiencias $u$ otro tipo de conocimientos no acordes con los programas en el ámbito universitario, y por ello los autores mencionados proponen que el papel de la investigación en didáctica de la física puede ser el de iniciar nuevas y mejores estrategias de enseñanza en las clases de teoría y problemas, así como en las prácticas de laboratorio, para controlar sus efectos tanto en el aprendizaje o comprensión de la física como en la motivación hacia la misma.

En concordancia con lo antes expuesto, se demanda la preparación de personas altamente capacitadas que asuman su rol como hombres de ciencia, sin embargo, como lo mencionara Rodríguez (2004: 4), en Venezuela, existe desde hace mucho tiempo una crisis educativa, donde en muchos casos se aplica una enseñanza descontextualizada de la realidad local y mundial predominando, entre muchos factores, la necesidad de formación y concientización de los docentes sobre sus responsabilidades con la sociedad.

Asimismo, existe en el estudiante del nivel universitario, la posibilidad de presencia de algunas deficiencias en cuanto los conocimientos básicos necesarios para iniciarse en un estudio superior de la física, lo cual pudiera 
ocasionar, de una forma $u$ otra en éste, dificultades para asimilar y procesar nuevas informaciones a niveles más profundos de conocimiento, aunado a ello el hecho que, quizás el profesor no se interese por diagnosticar la situación presente en el ambiente de clase conduciendo el proceso de enseñanza aprendizaje por la vía menos apropiada, hacia el fracaso formativo del estudiante.

En referencia a lo descrito, la Universidad Nacional Experimental "Rafael María Baralt", ubicada en el estado Zulia, no escapa a la problemática planteada, puesto que según la comparación de su índice de ingreso y egreso en la carrera Educación Matemática y Física en los últimos períodos, se muestra el bajo nivel de egresados, según información suministrada por el control de estudios de la misma Universidad, lo cual queda representado por 134 estudiantes cuando en el mismo tiempo hubo un ingreso de 236 personas, deduciéndose de esto la existencia de deficiencias de los participantes en el área de estudio, donde posiblemente no se estén alcanzando las competencias requeridas en la formación de los mismos por distintas razones, originando un bajo rendimiento académico.

De igual manera, de acuerdo con lo anterior, gran parte de los estudiantes participantes de la carrera matemática y física en la nombrada universidad presentan dificultades en los primeros niveles en el área de las físicas, así como quizás se estén manifestando en estos, problemas para relacionar los diversos contenidos de las asignaturas con las experiencias de la realidad. Tales deficiencias parecen persistir por los índices de egreso presentados, con lo cual se pueden estar ocasionando problemas de repitencia o deserción de los participantes en la carrera, o conduciéndose hacia una baja calidad en los profesionales que logren egresar, quienes pudieran no conseguir el ritmo adecuado en los estudios y por ende limitar el proceso de aprendizaje. 
En función a lo planteado cabe preguntarse:

¿Aplican los docentes estrategias constructivistas en el proceso de aprendizaje de la física en la carrera Educación Matemática y Física?

\section{Objetivo de la investigación}

Determinar las estrategias constructivistas aplicadas por el docente para el aprendizaje de la física en la carrera Educación Matemática y Física.

\section{Marco referencial}

\subsection{Estrategias de Enseñanza basadas en Enfoque Constructivista}

Para Álviarez y otros (2005: 12), las estrategias desde un enfoque constructivista involucran poner en marcha la frase "Aprender a Aprender" la cual significa enseñar a los estudiantes a volverse aprendices autónomos, independientes y autorreguladores, capaces de mejorar su proceso de aprendizaje, lo que implica la habilidad de reflexionar sobre las maneras de entender el aprendizaje, donde el individuo debe conducirse hacia un proceso intrínseco que le permita aplicar eficientemente un conjunto de procedimientos con la flexibilidad necesaria para adaptarse a diferentes situaciones problemáticas y ser transferidas a otros escenarios con características similares.

Asimismo, para los mencionados autores hablar de estrategias es referirse a un conjunto de procedimientos donde la ejecución de las mismos ocurre asociado con otros tipos de recursos y procesos cognitivos disponibles para cualquier aprendizaje y por ende, éstas constituyen una forma específica de organizar los recursos disponibles del docente para obtener resultados consistentes al realizar una determinada tarea y suelen ser de diversos tipos y cada una utiliza diferentes elementos para la consecución de sus fines.

En ese sentido, se entiende que estas estrategias serán las herramientas, procedimientos, pensamientos, conjunto de actividades y 
operaciones mentales utilizadas tanto por el docente como por el estudiante tomando en cuenta aquello que mejor conoce o domina para lograr la construcción conjunta del aprendizaje significativo.

En referencia a lo descrito, el estudiante de una asignatura con niveles de abstracción como la física requiere aprender de una manera estratégica, lo cual implica que éste:

a) Controle sus procesos de aprendizaje. b) Se dé cuenta de lo que hace. c) Capte las exigencias de la tarea y responda consecuentemente. d) Planifique y examine sus propias realizaciones, pudiendo identificar aciertos y dificultades. e) Emplee estrategias de estudios pertinentes para cada situación. f) Valore los logros obtenidos y corrija sus errores. (Díaz y Hernández, 2010a: 19).

De igual manera, en lo que respecta a las estrategias de aprendizaje en términos generales, Díaz y Hernández (2010b: 20), plantean que es necesario que el docente se encuentre totalmente definido en los siguientes puntos:

a) Son procedimientos. b) Pueden incluir varias técnicas, operaciones 0 actividades específicas. c) Persiguen un propósito determinado: el aprendizaje y la solución de problemas académicos y/o aquellos otros aspectos vinculados con ellos. d) Son más que los "hábitos de estudio" porque se realizan flexiblemente. e) Pueden ser abiertas (públicas) o reservadas (privadas). d) Son instrumentos socioculturales aprendidos en contextos de interacción con alguien que sabe más.

Por otra parte, según Carretero, (2009: 12), las estrategias didácticas desde una perspectiva constructivista deben de tener pensadas al menos situaciones como el contexto donde se van a desarrollar, las habilidades o competencias de los participantes en la creación del conocimiento, los estilos de procesamiento del conocimiento de los participantes, sus estilos de aprendizaje, las necesidades y motivaciones en torno al contenido a aprender y la eficacia de la estrategia frente a otras alternativas estratégicas. 


\subsection{Aprendizaje de la Física}

La Física como disciplina académica es una combinación de elementos de los tipos conceptuales, experimentales y abstractos que intentan responder al estudio de los componentes fundamentales del universo resumidos en materia y energía, por lo que el aprendizaje de esta ciencia siempre presentará importantes desafíos en todos los niveles de instrucción.

Ahora, para este estudio aprender se reduce a la construcción de conocimientos por parte de los estudiantes mediante la incorporación de los elementos que les ofrece el entorno en estrecha relación con los que desarrollan dentro del ambiente académico, de manera que, asociado a la física, el aprendizaje estará enmarcado en el alcance de competencias, desde una perspectiva conceptual, experimental y abstracta, durante el paso de los educandos por la disciplina, las cuales son requeridas para egresar satisfactoriamente de la misma.

En conformidad con lo anterior, Braga et al. (2011b: 2), plantean que los estudiantes que ingresan en el área de la física, deben alcanzar un conjunto de habilidades y destrezas las cuales se deben poseer para garantizar un proceso de enseñanza aprendizaje efectivo durante el desarrollo de la carrera universitaria y entre las cuales mencionan: capacidades generales en el área cognitiva, pensamiento y capacidad de razonamiento, el trabajo de laboratorio, la resolución de problemas, así como también el desarrollo de habilidades afectivas que permitan al educando una formación adecuada en la mencionada disciplina.

Por otra parte, Llamas (2008b: 16), manifiesta que uno de los aspectos principales para tener en cuenta en el aprendizaje de la física es la construcción conceptual, puesto que entre los problemas actuales en la enseñanza de la asignatura se encuentra la simplificación y modificación de conceptos que conllevan al desarrollo de errores conceptuales, lo cual sucede porque muchas veces se estructuran contenidos sin tomar en cuenta el nivel 
de desarrollo de los estudiantes.

En ese sentido, Braga et al. (2011c: 12), manifiestan que la gran mayoría del material de estudio a consultar por un estudiante de física corresponde a materiales escritos, por lo cual es necesario que éste presente cierta capacidad de comprensión de lectura, puesto que pueden mantener deficiencias de aprendizaje durante el período de estudio.

En el mismo orden de ideas, los autores anteriores destacan que un estudiante de la disciplina Física, debe tener la suficiente habilidad para representar la realidad ya sea a través de modelos, diagramas, gráficos, el uso de afirmaciones verbales así como de diversos símbolos y fórmulas, además de lograr una necesaria comprensión de teoremas, leyes, algoritmos, propiedades y conceptos, que va mucho más allá de una simple memorización de acciones y teorías, debido a que estas dificultades estarán presentes no sólo en todo su proceso de formación sino incluso cuando los mismos deseen ser formadores.

Asimismo, para Braga et al. (2011d: 15), en el desarrollo de cualquier curso de Física como de Ciencia en general, se debe hacer un uso frecuente del razonamiento lógico y el razonamiento de proporcionalidad; el primero para adquirir la habilidad de pasar de unas proposiciones a otras, partiendo de lo ya conocido o de lo que se cree conocer a lo desconocido o menos estudiado, donde el estudiante pueda darse cuenta cuando sus conclusiones están en total contradicción con la realidad; y el segundo para establecer algún tipo de relación considerando ciertos patrones comunes entre un hecho y otro, siendo imprescindible cuando se crean vínculos entre variables en las cuales se obtiene una constante de proporcionalidad, aplicada para expresar la relación entre cantidades.

\section{Marco metodológico.}

El estudio desarrollado corresponde al tipo descriptivo debido a que se 
busca describir una variable tal cual como se presenta en el momento de su medición. La investigación descriptiva según Hernández y otros (2010: 282), busca especificar las propiedades importantes de personas, grupos, comunidades o cualquier otro fenómeno que sea sometido a análisis. El estudio descriptivo mide o evalúa diversos aspectos, dimensiones 0 componentes del fenómeno o fenómenos a investigar.

Asimismo, en cuanto al diseño corresponde al de campo y documental. La investigación documental se realiza sobre la base de documentos o revisión bibliográfica, incluyéndose en esta categoría, entre otros, los diseños de modelos o propuestas. Según Chávez, (2012: 137), la finalidad de los estudios documentales es recolectar información a partir de documentos escritos y no escritos susceptibles de ser analizados y pueden clasificarse como investigaciones cualitativas o cuantitativas

La investigación de campo es el tipo de investigación que se apoya en informaciones que provienen entre otras, de entrevistas, cuestionarios, encuestas y observaciones. Esta investigación es de campo porque se desarrolló en el lugar en el cual se localizó el objeto de estudio y es documental, porque para su realización fue necesario revisar la bibliografía relacionada con la investigación.

Para la recolección de información en este estudio se hace uso de un cuestionario de respuestas policotómicas dirigido a los docentes que imparten la asignatura física dentro de la carrera Matemática y Física en la Universidad Nacional Experimental "Rafael María Baralt", ubicada en la ciudad de Cabimas, urbanización Los Laureles, Carretera H, Municipio Cabimas, Estado Zulia con el fin de identificar las estrategias constructivistas aplicadas por éstos en el proceso de aprendizaje de la física en la carrera Educación Matemática y Física, así como una Prueba Objetiva la cual fue destinada a los estudiantes del octavo semestre de la cátedra Física y Laboratorio V, del turno nocturno, Sede Cabimas del Programa Educación, Proyecto Educación Matemática y 
Física en la UNERMB, para caracterizar el proceso de aprendizaje de la Física desarrollado por los mismos en la mencionada carrera.

Para Camerano y Soubirón (2010: 18), las pruebas objetivas o de múltiple opción se componen de un conjunto de preguntas claras y precisas que requieren por parte del alumno, una respuesta breve, en general limitadas a la elección de una opción ya proporcionada. La prueba objetiva que se aplica en esta investigación consta de preguntas cerradas de selección simple, tomando en consideración para el análisis estadístico las respuestas; correcta o incorrecta, valoradas con los puntos 1 y 0 respectivamente.

Asimismo, los cuestionarios según Arribas, (2009: 12), son instrumentos utilizados para la recogida de información, diseñado para poder cuantificar y universalizar la misma y estandarizar el procedimiento de la entrevista. Su finalidad es conseguir la comparabilidad de esa información. En cuanto al cuestionario, el mismo consiste en un conjunto de ítems calificados mediante el método de escalamiento Lickert donde se solicita al sujeto externe su reacción eligiendo uno de los cinco puntos de la escala. A cada punto se le asigna un valor numérico, considerándose para este instrumento las alternativas: (Siempre), (Casi siempre), (pocas veces), (Casi nunca), (Nunca), valoradas de 5 a 1 punto en el orden respectivo.

\section{Análisis de los Resultados}

El análisis estadístico de los datos se realizó a partir de los resultados de la aplicación de un Cuestionario con una confiabilidad $r t t=0,97$ para la variable Estrategias Constructivistas y de una Prueba Objetiva para la variable Aprendizaje de la Física alcanzado una confiabilidad de KR20 =0,69

En ese sentido, se presenta un cuadro de distribución de frecuencias y porcentajes generales donde se determinan las estrategias constructivistas aplicadas por el docente en el proceso de aprendizaje de la Física, en la carrera Educación Matemática y Física, considerando como criterios para las 
alternativas los siguientes: (5.- Bastantes estrategias constructivistas. 4.Muchas estrategias constructivistas. 3.- Algunas estrategias constructivistas. 2.- Pocas estrategias constructivistas. 1.- Ninguna estrategia constructivista).

Tabla 1: Estrategias constructivistas aplicadas por el docente.

\begin{tabular}{|c|c|c|}
\hline ALTERNATIVA & $\mathbf{f i}$ & $\mathbf{f i} \%$ \\
\hline 5 & 92 & $33,82 \%$ \\
\hline 4 & 66 & $24,27 \%$ \\
\hline 3 & 50 & $18,38 \%$ \\
\hline 2 & 36 & $13,24 \%$ \\
\hline 1 & 28 & $10,29 \%$ \\
\hline
\end{tabular}

Fuente: El Autor (2017).

Gráfico 1: Estrategias constructivistas aplicadas por el docente.

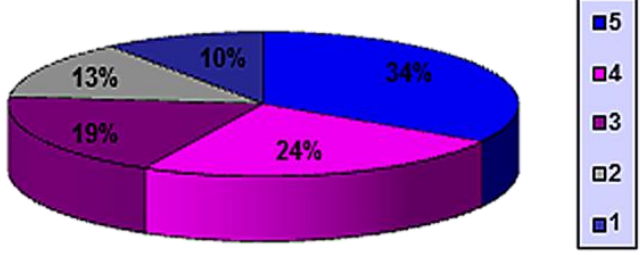

Fuente: El Autor (2017).

Haciendo referencia a las respuestas generales obtenidas para la variable Estrategias Constructivistas se demuestra que en sumatoria un total de $58,09 \%$ de los docentes que imparten la asignatura Física en la Universidad Nacional Experimental "Rafael María Baralt" están formados y utilizan estrategias sobre todo para propiciar el enlace entre los conocimientos previos y la nueva información que ha de procesar el estudiante, así como las aplican con alta frecuencia en el proceso de aprendizaje de la asignatura.

No obstante, existe un porcentaje relevante de la población estudiada que emplean en un rango muy menor e incluso nunca aplican estrategias en 
especial para el procesamiento de la información, además de no poseer una formación adecuada en el contexto donde se van a desarrollar las mismas y no consideran los estilos de procesamiento del conocimiento de los participantes en el momento de plantear alguna estrategia y esto queda representado por un total del $41,91 \%$.

En lo que se refiere a la variable aprendizaje de la Física, a continuación se presenta un cuadro de distribución de frecuencias y porcentajes generales para caracterizar el proceso de conocimiento alcanzado por el estudiante en la carrera Educación Matemática y Física, considerando como criterios para las alternativas los siguientes: (1.- Aprendizaje Significativo; 0.- Aprendizaje no Significativo).

Tabla 2: Aprendizaje de la Física alcanzado por el estudiante.

\begin{tabular}{|c|c|c|}
\hline ALTERNATIVA & fi & fi \% \\
\hline 1 & 146 & $34,76 \%$ \\
\hline 0 & 274 & $65,24 \%$ \\
\hline
\end{tabular}

Fuente: El Autor (2017).

Gráfico 2: Aprendizaje de la Física alcanzado por el estudiante.

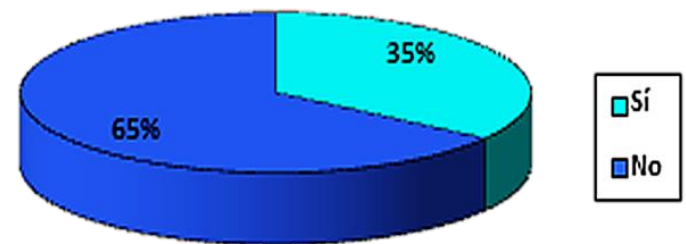

Fuente: El Autor (2017).

En función a los resultados generales obtenidos para la variable aprendizaje de la física, se observa en un porcentaje mayoritario del 65,24\% que los estudiantes cursantes de la asignatura Física y Laboratorio V, en la 
carrera Educación Matemática y Física no han alcanzado un aprendizaje significativo de la física en el transcurso de sus estudios en la Universidad Nacional Experimental "Rafael María Baralt", presentando deficiencias en las capacidades generales en el área cognitiva, las cuales involucran comprensión de material escrito, conexión entre realidad y representación, comprensión y formación de conceptos y descripción de acontecimientos desde distintos sistemas de referencia; así como en el desarrollo del razonamiento de proporcionalidad, lógico e hipotético deductivo, además del trabajo de laboratorio, puesto que no distinguen claramente entre observaciones e interpretaciones, entre otras cosas, en contraste con un $34,76 \%$ de la población estudiada que lo ha logrado.

\section{Conclusiones de la investigación}

Con relación a los objetivos propuestos en el estudio, los resultados estadísticos obtenidos, considerando además el análisis de resultados arrojados por los instrumentos y los aspectos teóricos que fundamentan el mismo, se pueden afirmar en términos generales las siguientes conclusiones sobre las estrategias constructivistas aplicadas por el docente para el aprendizaje de la física en la carrera Educación mención Matemática y Física.

En primer lugar, se evidencia que los docentes que imparten la asignatura física en la Universidad Nacional Experimental "Rafael María Baralt", aplican en su mayoría estrategias constructivistas en el proceso de enseñanza aprendizaje de la disciplina, variando las mismas en el momento de su uso, lo cual se refleja en un total del $58,09 \%$ de la población estudiada, no obstante existe en la institución un alto porcentaje de educadores que no emplean estrategias bajo este enfoque o lo hacen con muy poca frecuencia, y esto queda representado en un total del $41,91 \%$ de la generalidad investigada.

En segundo lugar, se observó que los docentes que imparten la asignatura física en la Universidad Nacional Experimental "Rafael María 
Baralt" utilizan, en una mayoría representada por un promedio del $75 \%$, estrategias para propiciar el enlace entre los conocimiento previos y la nueva información que ha de procesar el estudiante, su formación didáctica se basa principalmente en las habilidades o competencias de los participantes para la creación del conocimiento y la eficacia de la estrategia usada frente a otras alternativas estratégicas, así como aplican las mismas desde una perspectiva práctica.

En tercer lugar, se evidenció en un porcentaje mayoritario del $65,24 \%$ que los estudiantes cursantes de la asignatura Física y Laboratorio V, en la carrera Educación Matemática y Física no han alcanzado un aprendizaje significativo de la física en el transcurso de sus estudios en la Universidad Nacional Experimental "Rafael María Baralt", manifestando deficiencias tanto en las capacidades generales en el área cognitiva, en el pensamiento y capacidad de razonamiento y sobre todo en el trabajo de laboratorio.

En cuarto lugar, se demostró que los docentes que imparten la asignatura física en la Universidad Nacional Experimental "Rafael María Baralt", no aplican en su mayoría, las estrategias constructivistas más adecuadas en la enseñanza de la física con el fin de lograr en sus estudiantes aprendizajes significativos, del mismo modo que no toman en cuenta el contexto donde se van a desarrollar las mismas y los estilos de construcción del conocimiento de los educandos mencionados.

\section{Reflexiones Finales}

Incentivar a los docentes hacia el estudio de nuevas teorías educativas como el aprendizaje acelerado, programación psiconeurolingüística e inteligencia emocional, que puedan ser aplicadas en la elaboración de estrategias educativas innovadoras utilizadas en el aprendizaje de la física.

Mejorar la capacidad de equipos y dotación del Laboratorio de Física, del Programa de Educación de la UNERMB, al objeto de lograr la optimización 
en cuanto a la aplicación de las estrategias por parte de los docentes se muestra lo siguiente:

- La experimentación en el laboratorio es la instancia que obliga al estudiante a enfrentar situaciones que requieren la elaboración de modelos particulares del experimento que deben de estar enmarcados en un modelo físico más general.

- Son muchos los estudiantes que toman el laboratorio como lugar de estudio. Muchas veces es allí que los alumnos llegan con inquietudes distintas, como cosas que quieren probar, que no necesariamente son parte de los cursos curriculares, por ejemplo, cuestiones de relatividad, estudio de sonido de instrumentos musicales y otros.

- No son pocas las vocaciones docentes y de investigación que han encontrado en el laboratorio un ámbito que los incentiva y motiva.

- Para los profesores el laboratorio es el lugar de "sala permanente" donde se genera intercambio de información, coordinación de cursos, trabajos escritos y exámenes, discusión de temas didácticos y de la disciplina.

- Es importante que en la Universidad se cuente con un grupo de herramientas e instrumentos que propicien el desarrollo de proyectos investigativos en el área de las ciencias físicas, donde además se motive al estudiante a establecer conexiones entre la teoría aprendida y los conocimientos prácticos que puede construir mediante la experimentación.

- Asimismo, la Universidad debe establecer constantemente convenios con las empresas encargadas de proveer la instrumentación adecuada a la institución en el área de las ciencias físicas.

- Por otra parte, es necesario establecer un control de calidad de los instrumentos y del tipo utilizado, así como también de la preparación del 
docente en el manejo de los mismos.

\section{Referencias}

Álviarez, L, Guerreiro, Y, \& Sánchez, A. (2005). El uso de estrategias constructivistas por docentes de inglés con fines específicos. Revista Venezolana de Ciencias de Sociales, 21(47). Recuperado de: http://www.scielo.org.ve/scielo.php?script=sci arttext\&pid=S1012$\underline{15872005000200006}$

Arribas, M. (2009). Diseño y Validación de Cuestionarios. (2da ed.). vol. 5(17), 23-29. Madrid, España: Matronas Profesión.

Braga, L., Gallardo R., Calderón M., Morales J., \& Kling N. (2011a,b,c,d). Espectro de dificultades que presentan los alumnos que ingresan a la Universidad de Concepción en las carreras de ingeniería y licenciatura en física. Concepción, España. Recuperado de: http://fisicaparalavida.webnode.com.ar/news/espectro-de-dificultadesque-presentan-los-alumnos-que-ingresan-a-la-universidad-deconcepcion-en-las-carreras-de-ingenieria-y-licenciatura-en-fisica-/

Camerano S. \& Soubirón E. (2010). Diseño de Pruebas Objetivas. Unidad Académica de Educación Química. Monte Video, Uruguay.

Carretero, M. (2009). Constructivismo y Educación. (3ra. ed.). Buenos Aires, Argentina: Paidós.

Chávez, N. (2012). Introducción a la Investigación Educativa. (6ta de.) Maracaibo, Editorial Universal.

Díaz, F. \& Hernández, G. (2010a,b). Estrategias docentes para un aprendizaje significativo. Una interpretación constructivista. (3ra. ed.). México, D.F.: McGraw-Hill.

Hernández S, Fernández C. \& Batista P. (2010). Metodología de la Investigación. (5ta ed.). Editorial McGraw-Hill.

Llamas A. (2008a,b). Una visión estudiantil de la enseñanza de la física en 
el nivel superior. UAZ. México. Unidad Académica de Física. Rodríguez, T. (2004). Retos de la formación de docentes en Venezuela.

Revista Pedagógica, vol. 25, №. 73, págs. 03-12. ISSN: 0798-9792.

Soussan G. (2009). Enseñar las ciencias experimentales Didáctica y

Formación. Santiago de Chile. Publicado por la Oficina Regional de Educación de la UNESCO para América Latina y el Caribe. 


\section{Avilner Rafael Páez Pereira}

e-mail: profavilnerp@gmail.com

Nacido en Carora, estado Lara, Venezuela, el 24 de

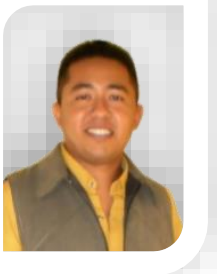

marzo de 1984. Cursó estudios de pregrado en la Universidad Nacional Experimental "Rafael María Baralt" (UNERMB), obteniendo a los 23 años el título de Lcdo. Educación Mención Integral graduado con la distinción CUM LAUDE, pero su pasión por las matemáticas lo llevó a cursar estudios de pregrado en esta área en la misma casa de estudios en paralelo con la maestría en Docencia para la Educación Superior en diferentes sedes, para obtener a los 28 años los títulos de Lcdo. en Educación Mención Matemática y Física y Magíster Scientiarum en Docencia para la Educación Superior siendo el primer lugar en ambas carreras. Posteriormente realizó el Doctorado en Educación en la Universidad Nacional Experimental "Rafael María Baralt" (UNERMB), estado Zulia en la espera del título hasta la fecha. En la actualidad se desempaña como Docente de aula por horas en el Liceo Bolivariano "José Véliz" y enlace de formación del circuito educativo $\mathrm{N}^{\circ} 17$ del municipio Torres, estado Lara con 11 años al servicio en el Ministerio del Poder Popular para la Educación.

El contenido de este manuscrito se difunde bajo una Licencia de Creative Commons ReconocimientoNoComercial-Compartirlgual 4.0 Internacional 